\title{
PRESENTACIÓN ATÍPICA DE DOLOR ABDOMINAL Y FIEBRE EN PACIENTE POSITIVO PARA COVID19. REPORTE DE CASO Y REVISIÓN DE LA LITERATURA
}

\author{
ATYPICAL PRESENTATION OF ABDOMINAL PAIN AND FEVER IN POSITIVE PATIENT FOR COVID19. \\ CASE REPORT AND LITERATURE REVIEW
}

Mauricio González-Arias ${ }^{1}$

\begin{abstract}
RESUMEN
Se presenta el caso clínico de una paciente femenino de 38 años de edad, vista en emergencia en un hospital de New York, que consulta por dolor abdominal, fiebre y astenia. Los estudios complementarios muestran linfopenia y leve alteración de transaminasas. Por TAC abdominopelvica, se visualiza imágenes en bases pulmonares, de tipo reticulonodular. Se le tomó muestra para COVID-19. Fue manejada en emergencias con hidratación parenteral, antipireticos, se le indica antibióticos por via oral y se instruye medidas sociales de aislamiento. Dos días después, consulta nuevamente, confirmadose COVID-19 positivo. Es seguida de manera ambulatoria, una semana después con buena evolución clínica. Adicionalmente se muestra una revisión clínica y explicación fisiopatológica del compromiso del tubo digestivo por el virus y la forma atípica de presentación con dolor abdominal.
\end{abstract}

Palabras clave: Dolor abdominal; COVID-19; Pandemia; Glicoproteína de la espiga del coronavirus; Infección por coronavirus (fuente: DeCS BIREME).

\begin{abstract}
The clinical case of a 38-year-old female patient, seen in an emergency room in a New York hospital, who consults for abdominal pain, fever and fatigue is presented. Complementary studies show lymphopenia and slight alteration of transaminases. Abdominopelvic CT scan visualizes images in the lung bases, reticulonodular type. A sample was taken for COVID-19. She was managed in emergencies with parenteral hydration, antipyretics, oral antibiotics are indicated and social isolation measures are instructed. Two days later, it cost again, confirming positive COVID-19. It is followed on an outpatient basis, one week later with good clinical evolution. Additionally, a clinical review and pathophysiological explanation of the involvement of the digestive tract by the virus and the atypical form of presentation with abdominal pain are shown.
\end{abstract}

Key words: Abdominal pain; COVID-19; Pandemic; Glycoprotein from the coronavirus spike; Coronavirus infection (source: MeSH NLM).

\section{REPORTE DE CASO CLÍNICO}

Paciente de 38 años del sexo femenino, sin antecedentes heredofamiliares de importancia, antecedentes quirúrgicos de colecistectomía hace 3 años, se presentó en nuestra sala de urgencias con las siguientes quejas: mialgias, dolor abdominal difuso y fiebre sin cuantificar. En la evaluación física inicial, los signos vitales eran: presión arterial: 110/70 mmHg, saturación de oxígeno: $98 \%$ al aire ambiente, frecuencia cardíaca: 120, temperatura: 39,6 grados Celsius.

La paciente tenía facies de dolor. A la auscultación, se notó incremento en los ruidos de peristalsis, y a la palpación superficial, doloroso en ambos cuadrantes inferiores. Se decidió llevar a cabo evaluación con 


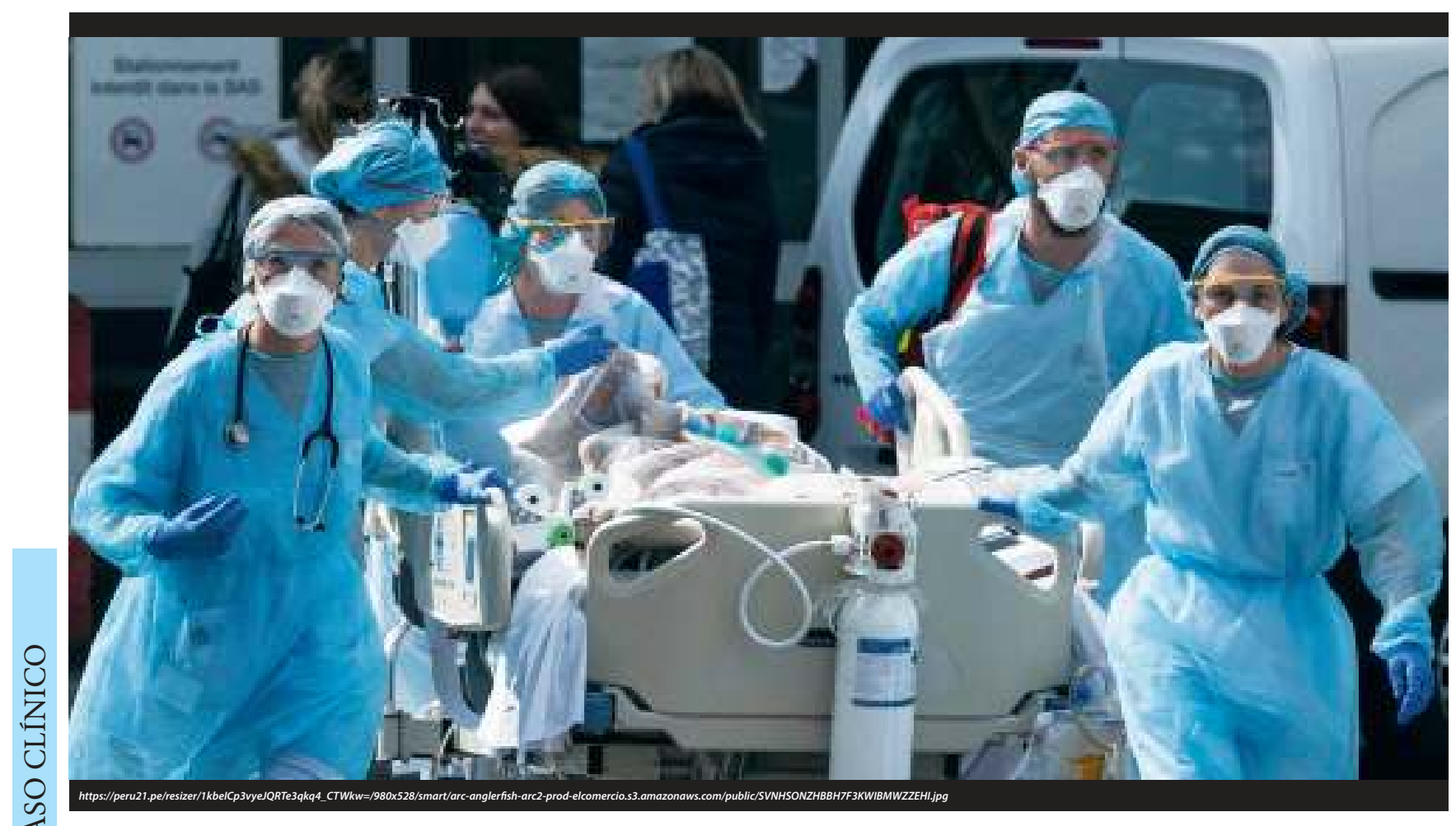

estudios paraclinícos y de imagenología con tomografia axial computarizada pélvica abdominal para descartar apendicitis aguda, diverticulitis, entre otras patologías de menor importancia.

Los estudios iniciales por citometría hemática, química sanguínea y perfil hepático se muestran en las Tablas 1 ,
2 y 3. La paciente había estado expuesta únicamente a miembros de la comunidad a través de su trabajo. Negó viajes a lugares geográficos que hasta esos momentos eran considerados endémicos para COVID19. También negó disuria y posteriormente, el análisis general de orina no mostró signos de patología de vías urinarias.

Tabla 1. Citometría hemática.

\begin{tabular}{lcc} 
& Resultado & Valores de referencia \\
\hline Glóbulos blancos & 6,26 & $4,30-11,00 \times 10(3) / \mathrm{mcl}$ \\
\hline Células rojas & 5,28 & $4,20-5,40 \times 10(6) / \mathrm{mcl}$ \\
\hline Hemoglobina & 15,7 & $12-16 \mathrm{~g} / \mathrm{dl}$ \\
\hline Plaquetas & 153 & $150-450 \times 10(3) / \mathrm{mcl}$ \\
\hline Neutrofilos\% & 74,1 & $50-65 \%$ \\
\hline Linfocitos \% & 18,1 & $25-40 \%$ \\
\hline Monocitos \% & 7 & $4,00-10,0 \%$ \\
\hline
\end{tabular}

Tabla 2. Química sanguínea.

\begin{tabular}{|c|c|c|}
\hline & Resultado & Valores de referencia \\
\hline Sodio & 137 & $136-145$ \\
\hline Potasio & 3,9 & $3,5-5,1$ \\
\hline Cloro & 101 & 98-107 \\
\hline Dióxido de carbono & 23 & $22-29$ \\
\hline Glucosa & 110 & 74-109 \\
\hline Nitrógeno ureico & 10 & $6-20$ \\
\hline Creatinina & 0,9 & $0,7-0-9$ \\
\hline Brecha aniónica & 13 & menor a 15 \\
\hline
\end{tabular}


Tabla 3. Perfil hepático.

\begin{tabular}{lcc} 
& Resultado & Valores de referencia \\
\hline Albúmina & 4,4 & $3,5-4,2 \mathrm{mg} / \mathrm{dl}$ \\
\hline Proteína total & 8,4 & $6,6-8,7 \mathrm{mg} / \mathrm{dl}$ \\
\hline Bilirubina total & 0,5 & $0,0-1,2 \mathrm{mg} / \mathrm{dl}$ \\
\hline Bilirubina directa & 0,01 & $0,0-0,3 \mathrm{mg} / \mathrm{dl}$ \\
\hline Fosfatasa alcalina & 94 & $35-104 \mathrm{U} / \mathrm{L}$ \\
\hline ALT & 55 & $0-31 \mathrm{U} / \mathrm{L}$ \\
AST & 47 & $0-32 \mathrm{U} / \mathrm{L}$ \\
\hline
\end{tabular}

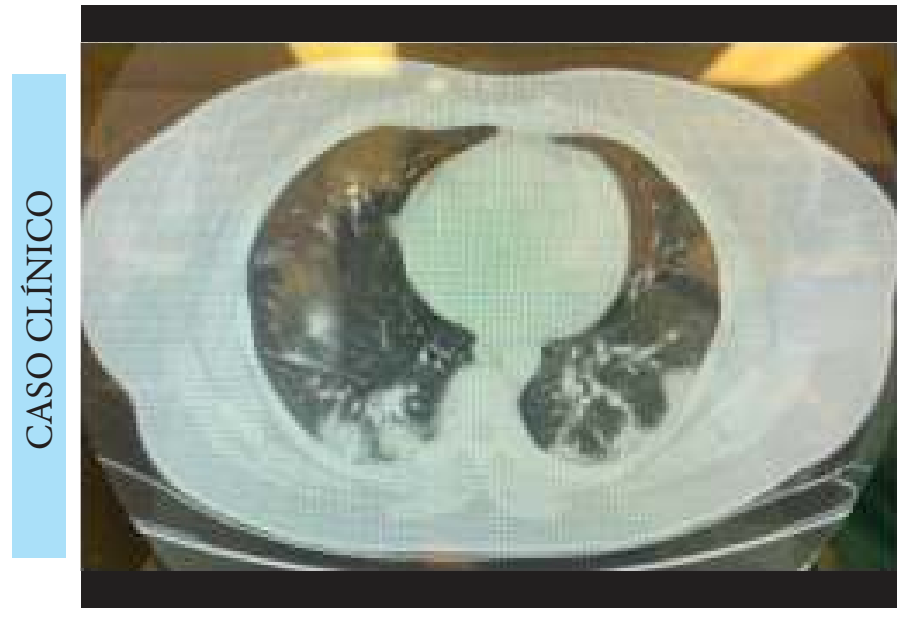

Figura 1. La imagen muestra corte de tomografía axial computarizada pélvico abdominal donde se aprecian bases de ambos pulmones.

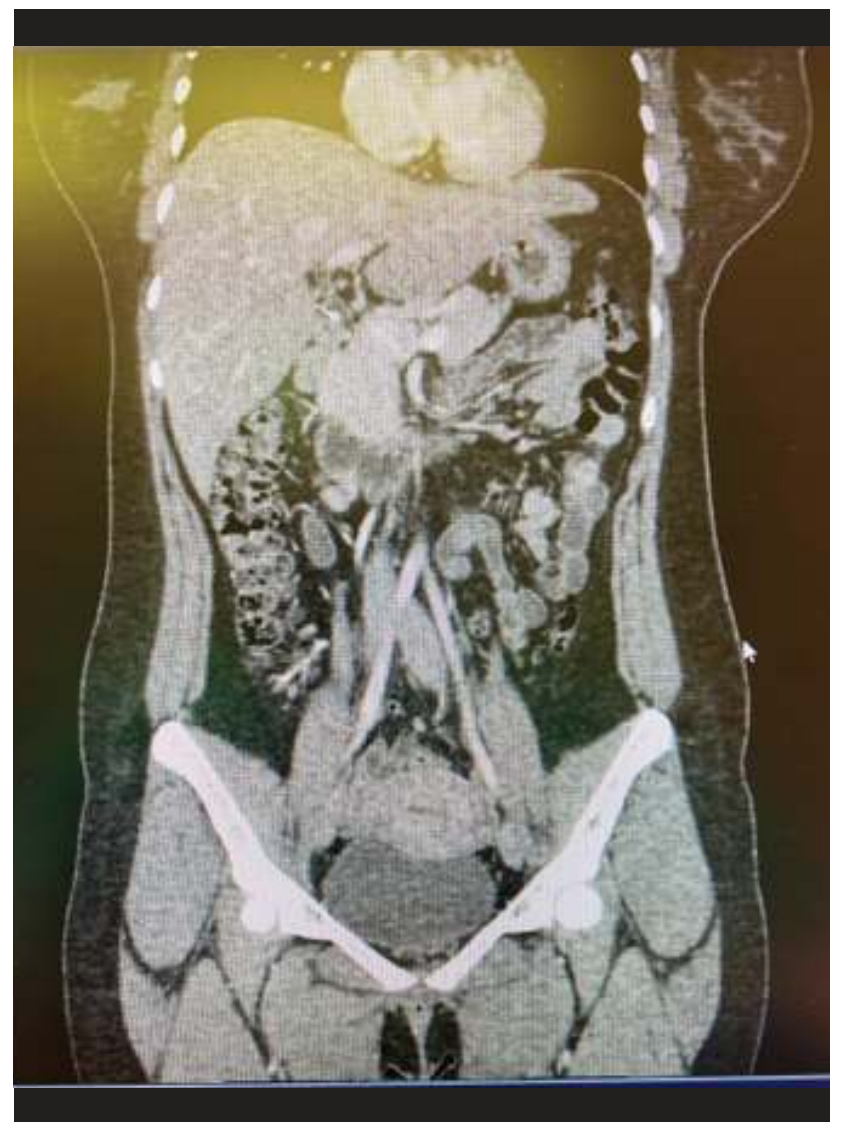

Figura 2. Muestra corte coronal de abdomen y pelvis.
El reporte radiológico describió hígado graso, apéndice normal, intestinos y estómago sin dilatación anormal o engrosamiento de pared. Infiltrados bilaterales en ambas bases pulmonares de apariencia reticulonodular, sugestivo de neumonía bilateral.

A la luz de linfopenia, alteración en transaminasas, fiebre y signos de neumonía bilateral, se decidió llevar a cabo la prueba de COVID19 con RT-PCR. La paciente fue dada de alta de la sala de emergencia con prescripción de moxifloxacino con el fin de tratar posible neumonía bacteriana. Además, fue estabilizada con líquidos intravenosos y tratada con antipiréticos. Se le proveyó educación sobre signos de alarma y aislamiento social.

A los dos días regresa a la sala de emergencia con falta de aire, fiebre y saturación de $97 \%$ sin oxígeno suplementario. Resultado de COVID19 fue positivo. Luciendo estable, y dado que se encontraba hemodinámicamente estable, fue dada de alta con estricto aislamiento social, continuación de antibiótico oral y fue seguida vía telefónica por una semana, teniendo desenlace óptimo con completa recuperación.

\section{DISCUSIÓN}

A finales de diciembre, 2019, un grupo de pacientes fueron admitidos a diferentes hospitales en China con diagnóstico de neumonía atípica de origen desconocido, y subsecuentemente, mediante trabajo de investigación epidemiológica, fueron vinculados a un mercado de venta de mariscos y animales vivos en Wuhan, provincia de Hubei, China ${ }^{(1)}$. Subsecuentemente, se encontró el causante viral de este brote, y se le nombró síndrome agudo respiratorio severo $\operatorname{Cov} 2$ (SARS Cov 2). En febrero, 11, la Organización Mundial de la Salud (OMS) nombró a la enfermedad causada por Sars Cov 2, COVID-19(2). Al momento de preparar este manuscrito, se han documentado mas de 660000 casos en el mundo(3).

Sars Cov 2 es un beta coronavirus de acuerdo a la OMS, donde el huesped primario son murciélagos ${ }^{(4)}$. Contiene una sencilla hebra de ARN y estudios genómicos han 
demostrado que es $80 \%$ parecido a SARS Cov 1 y $50 \%$ a $\mathrm{MERS}^{(5)}$.

La vasta mayoría de coronavirus contienen genes específicos dentro de las regiones codificantes ORF1 que codifican proteínas para la replicación viral, la nucleocápside y la formación de espigas ${ }^{(6)}$. Se ha demostrado que Sars Cov 2 gana entrada a la célula utiizando receptores enzima convertidora de angiotensina 2 (ECA2) ${ }^{(7)}$. Estos receptores se encuentran en múltiples tejidos, siendo de particular interés alveolos tipo 2 , enterocitos del intestino delgado y células de músculo liso en el endotelio vascular ${ }^{(8)}$.

El tiempo de incubación para COVID19 se cree que es entre dentro 14 días, posterior a lo exposición, y en varios reportes la mayoría de pacientes presenta síntomas 4-5 días después de exposición ${ }^{(9,10)}$. Las manifestaciones clínicas mas comunes reportadas hasta ahora son: fiebre, tos seca, fatiga, mialgias e incremento en la producción de esputo ${ }^{(11)}$.

Un estudio liberado por el centro de control de enfermedades en China llevado a cabo en 44,500 pacientes demostró que el $81 \%$ tuvo un curso leve (ausencia de neumonía), 14\% tuvo curso severo (disnea, hipoxia y mas del 50\% de infiltrados en imagen) y un estado crítico (fallo respiratorio, shock o falla orgánica mútiple) fue descrito en $5 \%$. La mortalidad total fue de $2,3 \%{ }^{(12)}$.

Interesantemente, en pacientes con diagnóstico de COVID 19 ha sido posible obtener Sars Cov 2 ARN en muestras de hisopo rectal ${ }^{(13)}$ y en muestras de heces fecales $^{(14)}$. un estudio llevado a cabo en 651 pacientes en Zhejiang, China, demostró que el 11,4\% de estos pacientes tenían al menos una queja del sistema gastrointestinal como náusea, vómito o diarrea ${ }^{(15)}$. En un grupo de cohorte de 140 pacientes positivos para COVID-19 en Wuhan, se documentó el porcentaje de quejas gastrointestinales: $24 \%$ experimentaron nausea, diarrea en un $18 \%$ y emesis en un $7 \%{ }^{(16)}$. No se reportó dolor abdominal.

En este caso presentamos a una paciente con queja principal de dolor abdominal y queremos compartir e instruir a médicos de primer nivel y especialistas en urgencias que a pesar de que COVID19 se manifiesta primordialmente en vias respiratorias, debido a la patofisiologia de Sars Cov 2, la sintomatología gastrointestinal puede estar presente en forma de vomito, diarrea y náusea, y aunque menos común, dolor abdominal.

Contribuciones de autoría: El autor participó en la génesis de la idea, diseño, recolección e interpretación de datos, análisis de resultados y preparación del manuscrito del presente artículo de investigación.

Financiamiento: Autofinanciado.

Conflicto de interés: El autor declara no tener conflicto de interés en la publicación de este artículo.

Recibido: 29 de marzo 2020

Aprobado: 02 de abril 2020

Correspondencia: Mauricio Gonzalez Arias.

Dirección: Metropolitan Hospital Center. New York Medical College, New York-EE.UU. Teléfono: 2124236262

Correo:drmauricio@drmauriciogonzalez.com

\section{REFERENCIAS BIBLIOGRÁFICAS}

1. Bogoch I. I, Watts A, Thomas-Bachli A, Huber C, Kraemer M. U, \& Khan K. Pneumonia of Unknown aetiology IN WUHAN, China: Potential for international spread via commercial air travel. J Travel Med 2020;27(2). p doi:10.1093/jtm/taaa008

2. Zhao S, Lin Q, Ran J, Musa S S, Yang G, Wang W, Wangr M. H. (2020). Preliminary estimation of the basic reproduction number of novel Coronavirus (2019-nCoV) in China, from 2019 to 2020: A data-driven analysis in the early phase of the outbreak. International Journal of Infectious Diseases 2020; Volume 92: 214-217. doi:10.1016/j.jijid.2020.01.050

3. Johns Hopkins coronavirus resource center. (n.d.). Retrieved March 29, 2020 from https://coronavirus.jhu.edu/map.html

4. Hui S, Azhar E. I, Madani,T. A, Ntoumi F, Kock R, Dar O, Petersen E. The continuing 2019-ncov epidemic threat of novel coronaviruses to global health - the Latest 2019 novel Coronavirus outbreak IN WUHAN, CHINA. 2020. International Journal of Infectious Diseases 2020; 91: 264-266. doi:10.1016/j. ijid

5. Lu Zhao X, Li J, Niu P, Yang B, Wu H, Tan W. Genomic characterisation and epidemiology of 2019 Novel CORONAVIRUS: Implications for VIRUS origins and receptor binding. The Lancet 2020; 395(10224): 565-574. doi:10.1016/ s0140-6736(20)30251-8

6. Boheemen S. V, Graaf M. D, Lauber C, Bestebroer T. M, Raj V. S, Zaki A. M, Fouchier R. A. Genomic characterization of a newly discovered coronavirus associated with acute respiratory distress syndrome in humans. mBio. 2012; (6) e00473-12. DOI: $10.1128 / \mathrm{mBio} .00473-12$

7. Zhou Yang X, Wang X, Hu B, Zhang L, Zhang W, Shi Z. A pneumonia outbreak associated with a new coronavirus of probable bat origin. Nature 2020 579(7798): 270-273. doi:10.1038/s41586-020-2012-7

8. Hamming I, Timens W, Bulthuis M, Lely A, Navis G, Goor H. V. Tissue distribution OF ACE2 protein, the functional receptor for SARS CORONAVIRUS. a first step in UNDERSTANDING SARS PATHOGENESIS. The Journal of Pathology 2020; 203(2): 631-637. doi:10.1002/path.1570

9. Lin, Huazhen and Liu, Wei and Gao, Hong and Nie, Jinyu and Fan, Qiao, Trends in Transmissibility of 2019 Novel Coronavirus-Infected Pneumonia in Wuhan and 29 Provinces in China (February 21, 2020). Available at SSRN: https://ssrn. com/abstract=3544821 or http://dx.doi.org/10.2139/ssrn.3544821

10. Guan W, Ni Z, Hu Y, Liang W, Ou C, He J, Zhong N. Clinical characteristics of Coronavirus DISEASE 2019 in China. New England Journal of Medicine 2020 doi:10.1056/nejmoa2002032

11. Wang D, Hu B, Hu C, Zhu F, Liu X, Zhang J, Peng,Z. Clinical characteristics of 138 hospitalized patients With 2019 Novel coronavirus-infected pneumonia IN WUHAN, CHINA. Jama 2020; 323(11): 1061. doi:10.1001/jama.2020.1585

12. Wu Z, Mcgoogan J M. Characteristics of and important lessons from THE Coronavirus DISEASE 2019 (COVID-19) outbreak in China. JAMA 2020; Published online. doi:10.1001/jama.2020.2648

13. Zhang W, Du R, Li B, Zheng X, Yang X, Hu B, Zhou, P. Molecular and serological investigation of 2019-NCOV infected patients: Implication of MULTIPLE Shedding routes. Emerging Microbes \& Infections 2020; 9(1): 386389. doi:10.1080/22221751.2020.1729071

14. Gu Han B, Wang, J. COVID-19: Gastrointestinal manifestations and Potential fecal-oral transmission. Gastroenterology 2020. doi:10.1053/j. gastro.2020.02.054

15. Zhang W, Du R, Li B, Zheng X, Yang X, Hu B, Zhou P. Molecular and serological investigation of 2019-NCOV infected patients: Implication of MULTIPLE Shedding routes. Emerging Microbes \& Infections 2020; 9(1): 386 389. doi:10.1080/22221751.2020.1729071

16. Zhang Dong $X$, Cao Y, Yuan Y, Yang Y, Yan Y, Gao Y. Clinical characteristics of 140 patients infected with Sars.cov.2 IN WUHAN, CHINA. Allergy 2020. doi:10.1111/all.14238 